\title{
Características clínico-epidemiológicas do câncer infantojuvenil no estado de Alagoas, Brasil
}

\author{
Clinical and epidemiological profile of childhood cancer in the state of Alagoas, Brazil
}

\author{
Claudio José dos Santos Júnior ${ }^{1}$, Cyndi Myrelle da Silva Barros Romão², Maria Jordana \\ Rocha Gomes Alves ${ }^{3}$, Arthur Moacir Costa Sampaio Batinga ${ }^{4}$, Vitória Mikaelly da Silva \\ Gomes $^{4}$, Nathalia Silva Araújo ${ }^{5}$, Roberta Fernandes Marinho ${ }^{6}$, Luiz Arthur Calheiros Leite ${ }^{7}$
}

Santos Júnior CJ, Romão CMSBR, Alves MJRG, Batinga AMCS, Gomes VMSG, Araújo NS, Marinho RF, Leite LAC. Características clínico-epidemiológicas do câncer infantojuvenil no estado de Alagoas, Brasil / Clinical and epidemiological profile of childhood cancer in the state of Alagoas, Brazil. Rev Med (São Paulo). 2018 set.out;97(5):454-60.

RESUMO: Objetivo: Avaliar os aspectos clínico-epidemiológicos do câncer infantojuvenil em Alagoas. Métodos: Trata-se de um estudo transversal, de caráter retrospectivo e quantitativo. As informações referentes aos diagnósticos de neoplasias infantojuvenis no Estado de Alagoas foram coletadas a partir de consulta aos prontuários dos centros de referência estaduais para tratamento de câncer na população de 0 a 19 anos. Resultados: Quanto ao perfil dos acometidos, a incidência maior foi em indivíduos do sexo masculino entre 5-9 anos, pardos, sem escolaridade, procedentes da $1^{\text {a }}$ região de saúde. As neoplasias mais frequentes foram: tumores hematológicos (leucemias e linfomas), tumores do sistema nervoso central, renais, ósseos, retinoblastoma e partes moles. A terapêutica mais empregada no tratamento dos casos foi a quimioterapia. Houve aumento na média de diagnósticos de casos novos de câncer infantojuvenil na população do estado de Alagoas. A letalidade do câncer infantojuvenil no período foi de 14,6\%. Conclusão: O câncer infantojuvenil em Alagoas, em concordância com o cenário nacional e internacional, continua sendo uma doença relativamente rara e de baixa incidência. $\mathrm{O}$ aumento dos casos verificado para o Estado pode estar associado a diversos fatores, entre estes, a maior capacitação profissional para triagem de sinais e sintomas clínicos, com consequente encaminhamento as unidades especializadas para investigação e diagnóstico precoce.

Descritores: Neoplasias/diagnóstico; Neoplasias/epidemiologia; Criança; Brasil/epidemiologia.

\begin{abstract}
Objective: The purpose of this study was to evaluate the clinical and epidemiological findings of childhood cancer in Alagoas. Methods: This is a cross-sectional, retrospective and quantitative study. All information related to the diagnosis of childhood neoplasms was collected from the records of Alagoas Oncology Centers. Results: The highest incidence was of males aged 5-9 years, brown, with no schooling, coming from the 1st health region. The most frequent neoplasms were: hematological tumors (leukemias and lymphomas), tumors of the central nervous system, renal, bone, retinoblastoma and soft tissues. The most used therapy in the treatment of the cases was chemotherapy. There was an increase in the average of diagnoses of new cases of childhood and adolescent cancer in the state of Alagoas. The lethality of childhood cancer in the period was $14.6 \%$. Conclusion: Childhood cancer in Alagoas remains to be a relatively rare and low incidence disease. The increase of the cases verified for the State may be associated to several factors, among them, the greater professional qualification for screening of clinical signs and symptoms, with consequent referral to specialized units for early diagnostic investigation.
\end{abstract}

Keywords: Neoplasms/diagnosis; Neoplasms/epidemiology; Child; Brazil/epidemiology.

Este trabalho foi realizado pelos membros do Projeto de Pesquisa "Rastreamento epidemiológico e diagnóstico precoce do câncer infantojuvenil em Alagoas" vinculado ao "Programa de diagnóstico precoce de câncer infantojuvenil" da UNCISAL.

1. Acadêmico da Faculdade de Medicina da Universidade Estadual de Ciências da Saúde de Alagoas. ORCID: http://orcid.org/0000-0002-2853-1968. E-mail: claudiosantosal@hotmail.com.

2. Enfermeira e Residente em Infectologia pela Universidade Estadual de Ciências da Saúde de Alagoas. ORCID: Romão CMSB - http://orcid.org/0000-00025134-0342; E-mail: cyndimyrelle94@gmail.com.

3. Acadêmica de Enfermagem da Universidade Estadual de Ciências da Saúde de Alagoas. ORCID: Alves MJRG - http://orcid.org/0000-0003-3390-0593. E-mail: jordana-rocha@hotmail.com.

4. Acadêmicos da Faculdade de Medicina da Universidade Federal de Alagoas. ORCID: Batinga AMCS - http://orcid.org/0000-0003-3901-5851; Gomes MS http://orcid.org/0000-0003-3785-7115. E-mail: arthur.sampaio.10@gmail.com, vitoriasnt.al@gmail.com.

5. Enfermeira. Santa Casa de Misericórdia de Maceió. http:/orcid.org/0000-0002-9795-1843. E-mail: nsa.1989@hotmail.com.

6. Psicóloga da Universidade Estadual de Ciências da Saúde de Alagoas. Coordenadora do Programa Diagnóstico Precoce do Câncer Infantojuvenil. Coordenadora de Projetos da Associação dos Pais e Amigos dos Leucêmicos de Alagoas - Apala. ORCID: http://orcid.org/0000-0001-8207-0210. E-mail: robertafernandes73@ gmail.com.

7. Professor Visitante da Universidade Estadual de Ciências da Saúde de Alagoas. Hospital Escola Dr. Helvio Auto. ORCID: http://orcid.org/0000-0002-97951843. E-mail: lahemato@yahoo.com.

Endereço para correspondência: Luiz Arthur Calheiros Leite. Residencial Jardim Europa, Quadra J 13, 150. Antares. Maceió, AL. CEP: 57048-148. E-mail: lahemato@hotmail.com. 


\section{INTRODUÇÃO}

Câncer em crianças e adolescentes é raro e precocemente. No entanto, ainda é umas das principais causas de óbito por doença nesta faixa etária ${ }^{1}$. O câncer infantojuvenil representa $1 \%$ de todas as neoplasias malignas no mundo. Nos Estados Unidos, a taxa de incidência anual média para todos os cânceres em indivíduos com menos de 20 anos é de 14,9 casos por 100.000 pessoas $^{2}$.

No Brasil, foram estimados 9.386 novos casos de câncer em crianças e adolescentes em 2010, e a mediana da taxa de incidência de câncer infantojuvenil foi de 15,4 casos por 100 mil habitantes ${ }^{3}$. Para o ano de 2018 são esperados um total de 12.500 novos casos de câncer em crianças e adolescentes no território nacional, com as regiões Sudeste e Nordeste apresentando os maiores números de casos novos, 5.300 e 2.900, respectivamente, seguidas pelas regiões Centro-Oeste (1.800 novos casos), Sul (1.300 novos casos) e Norte (1.200 novos casos $)^{4,5}$.

As neoplasias pediátricas mais comuns incluem as leucemias, os linfomas, os tumores do sistema nervoso central, neuroblastomas, tumores de Wilms, retinoblastomas, osteossarcomas e sarcomas ${ }^{5}$. Os aspectos morfológicos, a topografia e o comportamento clínico divergem dos tumores encontrados em adultos, pois geralmente, apresentam curto período de latência, rápido crescimento e são mais invasivos. Outro aspecto de extrema relevância clínica são as melhores respostas terapêuticas apresentadas ao tratamento ${ }^{6}$.

Uma média de $80 \%$ casos de câncer infantojuvenil podem obter a cura se forem tratados e diagnosticados precocemente ${ }^{4}$. Pautada nessa perspectiva, com a finalidade de garantir um melhor prognóstico ao paciente e ainda com o objetivo de diminuir a morbimortalidade por essas patologias, a política para o controle do câncer no Brasil evidencia a importância do diagnóstico precoce e dá destaque para a formação de recursos humanos atentos a essa estratégia.

No presente estudo objetivou-se realizar a caracterização clínico-epidemiológica das neoplasias infantojuvenis diagnosticadas nos três centros de referência de tratamento para o câncer infantojuvenis no Estado de Alagoas, Brasil.

\section{MÉTODOS}

Trata-se de um estudo descritivo, de corte transversal e abordagem quantitativa, realizado através de consulta ao acervo documental dos centros de referência estaduais para tratamento de neoplasias infantojuvenis no estado de Alagoas e da Apala - Associação dos Pais e Amigos dos Leucêmicos de Alagoas.

A população de estudo foi representada pelo universo de pacientes atendidos e tratados no âmbito do Sistema Único de Saúde nas unidades de oncopediatria da Santa Casa de Misericórdia de Maceió, Hospital Universitário Professor Alberto Antunes (HUPAA/UFAL) e Fundação Hospitalar da Agroindústria do Açúcar e do Álcool de Alagoas (Hospital do Açúcar).

Os critérios de inclusão no estudo foram: admissão nas unidades entre 1 de janeiro de 2013 e 31 de dezembro de 2016, possuir diagnóstico de câncer, idade inferior a 19 anos e possuir Ficha de Registro de Tumor devidamente preenchida constante no prontuário físico. Foram excluídos os pacientes com registros/dados incompletos no prontuário.

As informações necessárias para a realização do trabalho foram obtidas por meio da análise retrospectiva dos prontuários das crianças e adolescentes, tendo sido coletadas em formulário próprio e de acordo com os preceitos bioéticos preconizados pelo Conselho Nacional de Saúde, de omissão do nome do paciente e de outras informações que pudessem identificá-lo e/ou comprometer sua integridade física, psíquica social.

Os dados coletados incluíram informações sobre idade, sexo, raça/cor, naturalidade e informações relativas à doença, a exemplo do tipo e subtipo do câncer, ano do diagnóstico, opções terapêuticas e evolução do quadro.

Para o agrupamento dos casos foi empregada a $3^{a}$ edição da Classificação Internacional do Câncer na Infância da Sociedade Americana de Câncer, que divide o câncer em crianças e adolescentes em 12 grupos principais tomando como referência a morfologia e localização do tumor primário.

Os dados foram agrupados em banco de dados eletrônico através do Software Microsoft Office Excel 2017 e analisados por meio de estatística descritiva de frequência absoluta e relativa.

A pesquisa seguiu as recomendações da Resolução $n^{\circ}$ 466/2012 do Conselho Nacional de Saúde para estudos envolvendo seres humanos e obteve aprovação do Comitê de Ética em Pesquisa em Seres Humanos (CEP) da Sociedade de Ensino Superior de Alagoas/Faculdade Estácio de Sá, sob o parecer de número 2.103.683 (CAAE: 64846417.8.0000.5012).

\section{RESULTADOS}

A população do estudo constituiu-se de 314 casos novos de câncer em pacientes admitidos nos hospitais Santa Casa de Misericórdia de Maceió, HUPAA/UFAL e Hospital do Açúcar para tratamento de neoplasia infantojuvenil, no período de janeiro de 2013 a dezembro de 2016.

Ao longo desse período, dos pacientes de 0 a 19 anos diagnosticados com neoplasia, $182(58,0 \%)$ eram do sexo masculino e 132 (42,0\%) do sexo feminino (Tabela 1$)$.

Classificados de acordo com a cor da pele, 219 $(69,7 \%)$ pacientes eram pardos, $41(13,1 \%)$ eram brancos, 6 $(1,9 \%)$ eram negros, $2(0,6 \%)$ eram amarelos e $46(14,6 \%)$ não possuíam essa informação no prontuário.

A distribuição dos pacientes de acordo com a faixa etária foi a seguinte: 13 (4,1\%) eram menores de 1 ano, 
$77(24,5 \%)$ situavam-se na faixa etária de 1 a 4 anos, 87 $(27,7 \%)$ tinham de 5 a 9 anos, $56(17,8 \%)$ estavam na faixa etária de 10 a 14 anos e $81(25,9 \%)$ tinham de 15 a 19 anos (Tabela 1).

Tabela 1. Características clínico-epidemiológicas dos casos novos de câncer em pacientes de 0 a 19 anos no estado de Alagoas (2013-2016). $\mathrm{N}=314$

\begin{tabular}{|c|c|c|}
\hline Variável & $\mathbf{N}$ & $\%$ \\
\hline \multicolumn{3}{|l|}{ Ano do diagnóstico } \\
\hline 2013 & 71 & 22,6 \\
\hline 2014 & 82 & 26,1 \\
\hline 2015 & 86 & 27,4 \\
\hline 2016 & 75 & 23,9 \\
\hline \multicolumn{3}{|l|}{ Faixa Etária } \\
\hline$<1$ ano & 13 & 4,1 \\
\hline 1 a 4 anos & 77 & 24,5 \\
\hline 5 a 9 anos & 87 & 27,7 \\
\hline 10 a 14 anos & 56 & 17,8 \\
\hline 14 a 19 anos & 81 & 25,9 \\
\hline \multicolumn{3}{|l|}{ Sexo } \\
\hline Masculino & 182 & 58,0 \\
\hline Feminino & 132 & 42,0 \\
\hline \multicolumn{3}{|l|}{ Tratamento } \\
\hline Quimioterapia & 200 & 63.7 \\
\hline Radioterapia & 13 & 4.1 \\
\hline Cirurgia & 22 & 7.0 \\
\hline Quimioterapia + Radioterapia & 12 & 3.8 \\
\hline Quimioterapia + Cirurgia & 11 & 3.5 \\
\hline Radioterapia + Cirurgia & 1 & 0.3 \\
\hline Hormônioterapia & 1 & 0.3 \\
\hline Imunoterapia & 1 & 0.3 \\
\hline Outros & 14 & 4.5 \\
\hline Não iniciado / Sem registro & 39 & 12.4 \\
\hline \multicolumn{3}{|l|}{ Procedência (Região de Saúde) } \\
\hline $1^{\mathrm{a}} \mathrm{RS}$ & 142 & 45,2 \\
\hline $2^{\mathrm{a}} \mathrm{RS}$ & 10 & 3,2 \\
\hline $3^{\mathrm{a}} \mathrm{RS}$ & 22 & 7,0 \\
\hline $4^{\mathrm{a}} \mathrm{RS}$ & 24 & 7,6 \\
\hline $5^{\mathrm{a}} \mathrm{RS}$ & 22 & 7,0 \\
\hline $6^{\mathrm{a}} \mathrm{RS}$ & 11 & 3,5 \\
\hline $7^{\mathrm{a}} \mathrm{RS}$ & 39 & 12,4 \\
\hline $8^{\mathrm{a}} \mathrm{RS}$ & 17 & 5,4 \\
\hline $9^{\mathrm{a}} \mathrm{RS}$ & 13 & 4,2 \\
\hline $10^{\mathrm{a}} \mathrm{RS}$ & 14 & 4,5 \\
\hline
\end{tabular}

Fonte: Dados da Pesquisa. $* \mathrm{~N}$ da pesquisa $=314$, sendo $275 \mathrm{em}$ tratamento e 39 "Missing".

Quanto ao grau de formação, verificou-se que 87 $(27,60 \%)$ pacientes não possuíam nenhuma escolaridade, $129(41,30 \%)$ possuíam o ensino fundamental incompleto, $13(4,10 \%)$ tinham o ensino fundamental, $13(4,10 \%)$ possuíam nível médio e $1(0,3 \%)$ possuía nível superior incompleto; 71 pacientes $(22,6 \%)$ não possuíam essa informação registrada.

Analisando a incidência de câncer infantojuvenil nos 102 municípios do estado de Alagoas no quadriênio 2013-2016, verificou-se que 77 (75,5\%) municípios apresentaram registro pelo menos um caso de câncer infantojuvenil na população de 0 a 19 anos.

Ao agruparem-se os municípios por microrregiões de saúde, observou-se que a $1^{\mathrm{a}}$ mesorregião sanitária foi aquela que apresentou o maior número de casos (142; $45,2 \%$ ), totalizando dez cidades com registros (Barra de São Miguel, Flexeiras, Maceió, Marechal Deodoro, Messias, Paripueira, Pilar, Rio Largo, Santa Luzia do Norte, Satuba). A distribuição de todas as ocorrências para o período foi esquematizada na Figura 1.

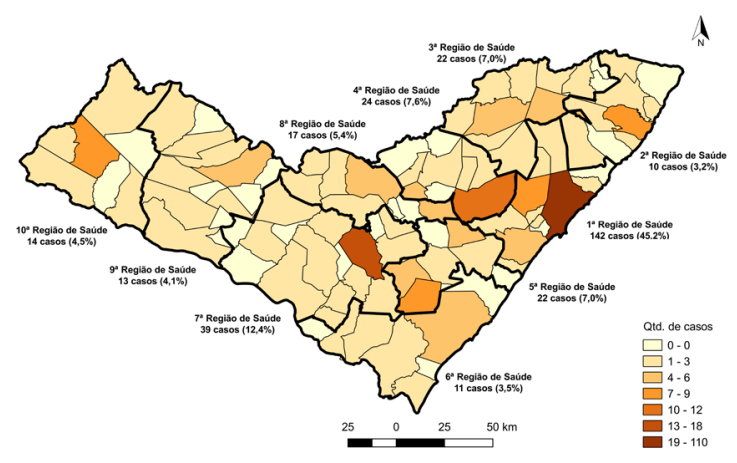

Fonte: Dados da Pesquisa.

Figura 1. Distribuição de casos novos de câncer infantojuvenil por região de saúde do estado de Alagoas, 2013-2016

As leucemias foram o tipo de câncer mais frequente, representando $36,0 \%$ dos diagnósticos no estado no período de 2013 a 2016. Os linfomas responderam por $17,8 \%$ dos casos e os tumores de SNC por 9,6\%.

Entre as leucemias, a leucemia linfoide aguda (LLA) foi a mais incidente, ocorrendo em $69,9 \%$ dos casos (79 pacientes). A leucemia mieloide aguda (LMA) foi responsável por 28,3\% dos diagnósticos (32 pacientes) e a leucemia mieloide crônica (LMC) ocorreu em apenas $1,8 \%$ (2 pacientes).

Entre os linfomas, o linfoma de Hodgkin foi responsável por $44,7 \%$ dos casos ( 25 pacientes), enquanto o linfoma não Hodgkin e o linfoma de Burkitt ocorreram em $32,1 \%$ (18 pacientes) e 7,1\% (4 pacientes), respectivamente; miscelânia de neoplasias linforreticulares e outros linfomas não especificados corresponderam a 16,1\% (9 pacientes).

Os tumores neuroectodérmicos (PNET) intracranianos e intraespinhais foram responsáveis por $70,0 \%$ dos casos de tumores do SNC (21 pacientes), enquanto que o ependimoma, o astrocitoma e outros gliomas ocorreram em $3,3 \%, 23,4 \%$ e $3,3 \%$ dos casos ( 1 , 7 e 1 pacientes), respectivamente. Quanto aos tumores do sistema nervoso simpático (SNS), o neuroblastoma ocorreu em $81,3 \%$ dos casos (13 pacientes), enquanto o ganglioneuroblastoma foi verificado em 18,7\% (3 pacientes). A Tabela 2 apresenta a frequência dos grupos de tumores infantojuvenis encontrados na população em estudo segundo a classificação proposta pela Sociedade Americana de Câncer. 
Santos Júnior CJ, et al. Características clínico-epidemiológicas do câncer infantojuvenil no estado de Alagoas,.

Tabela 2. Distribuição do número e percentagem dos casos novos de câncer em pacientes de 0 a 19 anos no estado de Alagoas, 20132016, segundo grupos e subgrupos da CICI-3

\begin{tabular}{|c|c|c|c|}
\hline GRUPOS E SUBGRUPOS & $\mathbf{N}$ & $\%$ (grupo) & $\%$ (total) \\
\hline I. LEUCEMIAS & 113 & & 36,0 \\
\hline Leucemia Linfoide Aguda & 79 & 69,9 & 25,2 \\
\hline Leucemia Mieloide Aguda & 32 & 28,3 & 10,2 \\
\hline Leucemia Mieloide Crônica & 2 & 1,8 & 0,6 \\
\hline II. LINFOMAS E NEO RETICULOENDOTELIAIS & 56 & & 17,8 \\
\hline Linfoma de Hodgkin & 25 & 44,7 & 8,0 \\
\hline Linfoma não Hodgkin (exceto Burkitt) & 18 & 32,1 & 5,7 \\
\hline Linfomas de Burkitt & 4 & 7,1 & 1,3 \\
\hline Miscelânia de Neoplasias Linforreticulares & 9 & 16,1 & 2,9 \\
\hline III. TUMOR DO SISTEMA NERVOSO CENTRAL (SNC) & 30 & & 9,6 \\
\hline Astrocitoma & 7 & 23,4 & 2,2 \\
\hline Ependimoma & 1 & 3,3 & 0,3 \\
\hline PNET intracraniano e intraespinhais & 21 & 70,0 & 6,7 \\
\hline Outros Gliomas & 1 & 3,3 & 0,3 \\
\hline IV. TU DO SISTEMA NERVOSO SIMPÁTICO (SNS) & 16 & & 5,1 \\
\hline Neuroblastoma & 13 & 81,3 & 4,1 \\
\hline Ganglioneuroblastoma & 3 & 18.7 & 1,0 \\
\hline V. RETINOBLASTOMA & 10 & & 3,2 \\
\hline Retinoblastoma & 10 & 100,0 & 3,2 \\
\hline VI. TUMORES RENAIS & 16 & & 5.1 \\
\hline Tumor de Wilms & 16 & 100,0 & 5,1 \\
\hline VII. TUMORES HEPÁTICOS & 5 & & 1,6 \\
\hline Hepatoblastoma & 2 & 40,0 & 0,6 \\
\hline Hepatocarcinoma & 3 & 60,0 & 1,0 \\
\hline VIII. TUMORES ÓSSEOS MALIGNOS & 15 & & 4,8 \\
\hline Osteossarcoma & 9 & 60,0 & 2,9 \\
\hline Condrossarcoma & 4 & 26,7 & 1,3 \\
\hline Sarcoma de Ewing & 2 & 13,3 & 0,6 \\
\hline IX. SCM DE PARTES MOLES (SPM) & 8 & & 2,5 \\
\hline Rabdomiossarcoma & 4 & 50,0 & 1,3 \\
\hline Fibrossarcoma & 1 & 12,5 & 0,3 \\
\hline Outros SPM Especificados & 3 & 37,5 & 1,0 \\
\hline X. TUMORES DE CÉLULAS GERMINATIVAS (TCG) & 14 & & 4,5 \\
\hline TCG Extracranianos e Extragonadais & 1 & 7,1 & 0.3 \\
\hline TCG Gonadais & 13 & 92,9 & 4.1 \\
\hline XI. CARCINOMAS E OUTRAS NEO EPITELIAIS & 21 & & 6,7 \\
\hline Carcinoma de Tireoide & 6 & 28,6 & 1,9 \\
\hline Carcinoma de Nasofaringe & 4 & 19,0 & 1,3 \\
\hline Melanoma maligno & 1 & 4,8 & 0,3 \\
\hline Carcinoma de Pele & 4 & 19,0 & 1,3 \\
\hline Outros carcinomas e carcinomas não especificados & 6 & 28,6 & 1,9 \\
\hline XII. OUTRAS NEO NAO ESPECIFICADAS & 10 & & 3,2 \\
\hline Outros TU Malignos não especificados & 10 & 100,0 & 3,2 \\
\hline TODOS OS GRUPOS COMBINADOS & 314 & - & 100,0 \\
\hline
\end{tabular}

LEGENDA: CICI-3: Classificação Internacional do Câncer Infantojuvenil $3^{a}$ versão.

Fonte: Dados da Pesquisa. 
Analisando-se o tipo de terapêutica recebida pelos pacientes, verificou-se que a quimioterapia foi utilizada no tratamento de 200 pacientes $(63,7,7 \%)$, a radioterapia em 13 pacientes $(4,1 \%)$ e a cirurgia foi realizada em 22 pacientes (7,0\%). A combinação desses tratamentos ocorreu 24 casos (7,6\%). As aplicações de hormônioterapia e imunoterapia foram as técnicas menos empregadas, com apenas $1(0,3 \%)$ registro de aplicação para cada.

Outras estratégias foram realizadas em 14 pacientes $(5,1 \%)$, aqui entendidos como sendo aqueles métodos complementares usados junto ao atendimento médicohospitalar regular, excetuando-se os tratamentos elencadas na Tabela 1.

$\mathrm{Na}$ análise dos prontuários, verificou-se também que, quando da coleta dos dados, 39 pacientes estavam fora de tratamento e/ou não possuíam essa informação registrada nos documentos analisados.

Tabela 3. Letalidade (\%) das neoplasias infanto-juvenil diagnosticadas no estado de Alagoas, segundo o tipo de câncer (2013-2016)

\begin{tabular}{lccc}
\hline Tipo de câncer & Casos & Óbitos & Letalidade \% \\
\hline LLA & 79 & 14 & 17,7 \\
LLM & 32 & 11 & 34,4 \\
PNET & 21 & 5 & 23,8 \\
Osteossarcoma & 9 & 3 & 33,3 \\
Astrocitoma & 7 & 2 & 28,6 \\
Neuroblastoma & 13 & 2 & 15,4 \\
Rabdomiossarcoma & 4 & 2 & 50,0 \\
Ependimoma & 1 & 1 & 100,0 \\
Hodgkin & 25 & 1 & 4,0 \\
Não-Hodgkin & 18 & 1 & 5,6 \\
Neoplasias & 9 & 1 & 11,1 \\
Linforreticulares & 2 & 1 & 50,0 \\
Ewing & 16 & 1 & 6,3 \\
Tumor de Wilms & 10 & 1 & 10,0 \\
Outros tumores & & &
\end{tabular}

Fonte: Dados da Pesquisa.

Ao longo do período de 2013 a 2016, ocorreram 46 óbitos (14,6\%) pelas causas em estudo. A letalidade dos principais tipos de tumores infantojuvenis também pode ser visualizada na Tabela 3.

Tabela 4. Casos óbito por neoplasia infanto-juvenil no estado de Alagoas, segundo a faixa-etária (2013-2016)

\begin{tabular}{lcc}
\hline Faixa etária & N & Óbitos \\
\hline menor de 1 ano & 5 & 10,9 \\
1 a 4 anos & 14 & 30,4 \\
5 a 9 anos & 8 & 17,4 \\
10 a 14 anos & 9 & 19,6 \\
14 a 19 anos & 10 & 21,7 \\
Total & $\mathbf{4 6}$ & $\mathbf{1 0 0 , 0}$ \\
\hline
\end{tabular}

A distribuição dos óbitos por câncer infantojuvenil, de acordo com a faixa etária, foi a seguinte: 5 (10,9\%) eram menores de 1 ano, $14(30,4 \%)$ situavam-se na faixa etária de 1 a 4 anos, $8(17,4 \%)$ tinham de 5 a 9 anos, $9(19,6 \%)$ estavam na faixa etária de 10 a 14 anos e $10(21,7 \%)$ tinham de 15 a 19 anos. As maiores frequências de óbitos ocorreram nas faixas etárias de 1 a 4 anos $(30,4 \%)$ e de 14 a 19 anos (21,7\%). A Leucemia Linfoide Aguda apresentou a maior frequência de óbitos. Os números referentes à letalidade por tipo de neoplasia infantojuvenil apresentou grande variação.

Investigando-se a história de câncer no grupo de parentes dos pacientes, verificou-se que em 21 prontuários $(6,7 \%)$ constava a "presença de câncer no grupo familiar", ao passo que 77 (24,5\%) não tinham história prévia de neoplasias na família. Na maioria dos prontuários (216 pacientes, o que corresponde a 68,8\%) essa informação não estava registrada.

\section{DISCUSSÃO}

O câncer pediátrico continua sendo uma doença de grande preocupação para a saúde pública constituindo-se na primeira causa de mortalidade na faixa etária de 1 a 19 anos. ${ }^{4}$ Representa $8 \%$ dos óbitos em crianças e adolescentes e $2 \%$ de todas as neoplasias malignas no Brasil ${ }^{1}$.

Mundialmente, utiliza-se a Classificação Internacional do Câncer na Infância (International Classification of Childhood Cancer), criada em 1996 e hoje em sua terceira edição, para agrupamento dessas doenças. Tal classificação toma como referência as novas classificações morfológicas propostas na CID-O-3 (2000) 7 .

Em trabalho publicado no ano de 2016 verificou-se que a incidência do câncer pediátrico no mundo de acordo com sexo foi de 232,31 por milhão no sexo feminino e 218,07 por milhão para o sexo masculino, com faixa etária de menor incidência entre 15 a 19 anos. ${ }^{8}$ Park et al, mostraram que crianças de 1 a 4 anos de idade tiveram as maiores taxas de incidência. ${ }^{9}$ A incidência do câncer pediátrico pelo mundo é similar aos dados de estudos do Brasil, mostrando que esses dados podem ser usados como taxas de incidência de câncer de infância e adolescência no território nacional em futuros estudos epidemiológicos ${ }^{5,8}$.

Em um trabalho de revisão levantou-se a frequência do câncer infantojuvenil Brasil. Os autores verificaram que as leucemias agudas representaram $30 \%$ de todos os casos de câncer na faixa etária, seguida dos tumores do SNC $(17 \%$ a $25 \%)$. Os tumores renais correspondiam a cerca de $10 \%$ dos tumores pediátricos, com o tumor de Wilms representando $95 \%$ destas últimas. O quarto tumor mais frequente foi o retinoblastomas ${ }^{10}$.

Pollock et al. ${ }^{11}$ e Rodrigues et al. ${ }^{12}$ demonstraram com dados americanos e brasileiros, respectivamente, os tumores pediátricos que mais eram diagnosticados nos serviços. Tanto no estudo de Atlanta como no Hospital do 
Câncer de São Paulo as leucemias, os linfomas, o tumor de Wilms, o neuroblastoma, os tumores do SNC, os tumores ósseos e de partes moles figuraram como as neoplasias mais comuns.

Camargo et al..$^{5}$ realizaram a primeira análise descritiva da incidência de câncer infantojuvenil no Brasil tendo como referencial os dados de incidência de neoplasias em crianças e adolescentes em 14 cidades brasileiras que possuíam implementado o Registro de Câncer Base População Brasileira (RCBP). Neste estudo a cidade de São Paulo apresentou o maior número de casos de câncer infantojuvenil com 3.667 diagnósticos e a cidade com menor taxa de detecção foi Jaú (SP), com registro de 29 casos. Na região nordeste, Fortaleza apresentou 587 casos, seguido de Recife; o menor número de ocorrências no Nordeste ocorreu em Aracaju ( $n=123$ casos $)^{5}$. A cidade de Maceió, bem como demais cidades do estado de Alagoas, não foram incluídas neste estudo, provavelmente por não possuírem consolidado o RCBP. No geral, a pesquisa identificou que os tipos de neoplasias mais comuns na faixa etária foram as leucemias (18 a 41\%), linfomas (13 a 24\%) e tumores do SNC (7 a 17\%).

Nosso estudo mostrou similar distribuição dos tumores infantojuvenis, com as leucemias sendo as neoplasias mais frequentes (36\%), seguidas pelos linfomas e neoplasias reticuloendoteliais $(17,8 \%)$, tumores do SNC $(9,6 \%)$, tumores do $\operatorname{SNS}(5,1 \%)$, retinoblastoma $(3,2 \%)$, tumores renais $(5,1 \%)$, tumores hepáticos $(1,6 \%)$, tumores ósseos malignos $(4,8 \%)$ sarcomas de partes moles $(2,5 \%)$, tumores de células germinativas $(4,5 \%)$, carcinomas e neoplasias epiteliais $(6,7 \%)$ e outras neoplasias não especificadas $(3,2 \%)$.

Nosso estudo também mostrou que a quimioterapia é o tratamento antineoplásico mais utilizado para os pacientes da pesquisa $(72,7 \%)$ e também descrito na literatura como principal tratamento para o câncer.

Os dados eletrônicos do Registro Hospitalar de Câncer (RHC/INCA) revelam que a média de diagnósticos de casos novos de câncer infantojuvenil na população do estado de Alagoas era de 58 casos/ano para o período de $2009-2010^{13}$. No presente estudo, observou-se que entre 2013-2016 a média de diagnósticos foi de 78,5 casos/ano.

O aumento de casos nessa faixa etária pode estar associado a diversos fatores, entre eles a capacitação de profissionais da atenção básica do interior do Estado para reconhecimento dos sinais e sintomas do câncer infantojuvenil e consequente encaminhamento para os serviços especializados em oncologia e hematologia da cidade de Maceió. Como na maioria das capitais brasileiras existe uma tendência de centralização dos casos diagnosticados nas grandes capitais, isso pode levar a um viés de distribuição entre as regiões, podendo ser considerada uma limitação dos estudos que trabalham na perspectiva de especializar a ocorrência de eventos em saúde, como é o caso do presente trabalho.

A atuação em áreas-chave como a prevenção nos níveis primário (promoção da saúde) e secundário (detecção do surgimento da doença nos estágios iniciais) e melhor preparação de profissionais da atenção básica para suspeitar dos sinais e sintomas e encaminhar para triagem oncológica as crianças e adolescentes, têm demonstrado serem ferramentas efetivas de investimento em diagnóstico e manejo do câncer infantojuvenil, sendo tais corroboradas pela Política Nacional de Controle do Câncer ${ }^{14,15}$.

Em Alagoas, o estímulo à detecção precoce do câncer infantojuvenil vem sendo trabalhado desde 2008 por meio da implementação do Programa do Diagnóstico Precoce do Câncer Infantojuvenil da Universidade Estadual de Ciências da Saúde de Alagoas (Uncisal) e da Apala (Associação dos Pais e Amigos dos Leucêmicos de Alagoas) em parceria com o Instituto Ronald McDonald. Este programa tem como objetivo capacitar equipes da Estratégia Saúde de Família (ESF) dos municípios do estado de Alagoas para suspeitar dos sinais e sintomas da doença nas crianças e adolescentes e encaminhar para diagnóstico diferencial em curto espaço de tempo. Outras ações do programa incluem o desenvolvimento de eventos e projetos, com ações educativas e informativas destinadas à capacitação profissional de estudantes da área de saúde, com a mesma finalidade. Até 2018 foram capacitados cerca de 3.600 profissionais de vários municípios alagoanos por meio da execução das capacitações oferecidas às equipes de atenção básica, impactando em 25.149 crianças e adolescentes de áreas cobertas pela ESF.

No âmbito nacional, um estudo de avaliação do impacto do Programa Diagnóstico Precoce do Câncer Infantojuvenil feito em 2013 pelo Instituto Fernandes Figueira IFF/NATS/Fiocruz apresentou como principais resultados do programa um aumento de $23 \%$ dos casos e $61 \%$ de redução do tempo de trajetória do paciente desde o início dos sinais e sintomas ao início do tratamento no país $^{14,15}$.

Convém ressaltar que a capital do Estado (Maceió) faz parte da primeira regional de saúde, centralizando todos os centros de tratamento para oncopediatria de Alagoas. Dada a distância territorial dos municípios localizados nas regiões $9^{\circ}$ e $10^{\circ}$ regiões de saúde, por estas se localizarem no extremo oposto do estado, esta realidade pode comprometer o acesso ao tratamento ou ainda levar um percentual de casos a buscarem assistência no estado vizinho, sendo relevante considerar que é possível haver uma demanda reprimida ou a migração desses pacientes para estados vizinhos.

Devido à ausência de informações sistematicamente analisadas sobre o quadro atual do câncer infantojuvenil no Estado do Alagoas, este estudo abre uma perspectiva para a realização de novos trabalhos apresentando uma descrição da situação epidemiológica e da atenção a esta patologia. Este trabalho também provocou o desejo de realizar análises clínicas e moleculares para estudar a correlação com o estadiamento dos tumores pediátricos e a sobrevida dos pacientes. 


\section{CONCLUSÃO}

O câncer infantojuvenil em Alagoas apresenta concordância com o cenário nacional e internacional e continua sendo uma doença relativamente rara e de baixa incidência.

Houve maior incidência de casos em indivíduos do sexo masculino com idades entre 5-9 anos, pardos e sem escolaridade, sendo as neoplasias mais frequentes tumores hematológicos (leucemias e linfomas), tumores do sistema nervoso central, renais, ósseos, retinoblastoma e partes moles. A terapêutica mais empregada no tratamento foi a quimioterapia.
Evidenciou-se, ainda, que houve maior incidência de neoplasias infantojuvenis na $1^{\mathrm{a}}$ região sanitária, fato que pode estar associado a concentração de serviços especializados nesta área.

Acredita-se que a capacitação dos profissionais da atenção básica para triagem de casos suspeitos e direcionamento aos serviços especializados em oncohematologia pode estar relacionada ao aumento no número de diagnósticos, que passou 58 casos/ano no período de 2009-2010 para 78,5 casos/ano entre 2013-2016.

AGRADECIMENTOS: Pelo suporte estrutural, agradecemos as seguintes instituições: Santa Casa de Misericórdia de Maceió, Hospital Universitário Professor Alberto Antunes, Fundação Hospitalar da Agroindústria do Açúcar e do Álcool de Alagoas, Associação dos Pais e Amigos dos Leucêmicos de Alagoas e Universidade Estadual de Ciências da Saúde de Alagoas.

CONFLITOS DE INTERESSE: Não existem conflitos de interesse.

PARTICIPAÇÃO DOS AUTORES: Claudio José dos Santos Júnior: Coleta de dados, redação do artigo, análise de dados, elaboração das tabelas e gráficos, revisão final. Cyndi Myrelle da Silva Barros Romão: Coleta de dados, redação do artigo, análise e revisão do manuscrito final. Maria Jordana Rocha Gomes Alves: Coleta de dados e redação do artigo. Arthur Moacir Costa Sampaio Batinga: Coleta, sistematização e tabulação de dados, redação do artigo. Vitória Mikaelly Da Silva Gomes: Coleta e tabulação de dados. Nathalia Silva Araújo. Orientação na elaboração do projeto. Escrita do artigo. Roberta Fernandes Marinho: Elaboração do projeto, coleta de dados, redação do artigo final e revisão geral. Luiz Arthur Calheiros Leite: Orientação na elaboração do projeto, análise de dados, revisão do texto e edição, discussão dos achados, submissão do artigo.

FOMENTO: UNCISAL/APALA.

\section{REFERÊNCIAS}

1. Grabois MF, Oliveira EXG, Carvalho MS. Childhood cancer and pediatric oncologic care in Brazil: access and equity. Cad. Saúde Pública. 2011;27(9):1711-20. doi: https://doi. org/10.1590/S0102-311X2011000900005.

2. Malogolowkin MH, Quinn JJ, Steuber CP, Siegel SE. Clinical assessment and differential diagnosis of the child with suspected cancer. In: Pizzo PA, Poplack DG, editors. Principles \& practice of pediatric oncology. 5th Ed. Philadelphia: Lippincott Williams \& Wilkins; 2006. p.165-81.

3. Brasil. Ministério da Saúde. Instituto Nacional de Câncer José Alencar Gomes da Silva (INCA). Estimativa 2012: incidência de câncer no Brasil. Rio de Janeiro: INCA; 2011 [citado 11 jul. 2018]. Disponível em: https://www.inca.gov.br/.

4. Brasil. Ministério da Saúde. Instituto Nacional de Câncer José Alencar Gomes da Silva (INCA). Estimativa 2018: incidência de câncer no Brasil. Rio de Janeiro: INCA; 2017 [citado 11 jul. 2018]. Disponível em: https://www.inca.gov.br/.

5. Camargo B, Oliveira SM, Rebelo MS, Reis RS, Ferman S, Noronha $\mathrm{CP}$, et al. Cancer incidence among children and adolescents in Brazil: first report of 14 population-based cancer registries. Int J Cancer. 2010;126(3):715-20. doi: https://doi.org/10.1002/ijc.24799

6. Steliarova-Foucher E, Colombet M, Ries LAG, Moreno F, Dolva A, Bray F, et al. International incidence of childhood cancer, 2001-10: a population-based registry study. Lancet Oncol. 2017;18(6):719-31. doi: https://doi.org/10.1016/ S1470-2045(17)30186-9.

7. Steliarova-Foucher E, Stiller C, Lacour B, Kaatsch P. International Classification of Childhood Cancer, third edition. Cancer. 2005;103(7):1457-67. doi: https://doi.org/10.1002/ cncr.20910.

8. Balmant NV, Reis RS, Oliveira JFP, Ferman S, Santos MO,
Camargo B. Cancer Incidence Among Adolescents and Young Adults (15 to 29 Years) in Brazil. J Pediatr Hematol Oncol. 2016;38(3):e88-e96. doi: https://doi.org/10.1097/ MPH.0000000000000541.

9. Parkin DM, Stiller CA, Draper GJ, Bieber CA. The international incidence of childhood cancer. Int $\mathrm{J}$ Cancer. 1988;42:511-20. doi: https://doi.org/10.1002/ijc.2910420408.

10. Rodrigues KE, Camargo B. Diagnóstico precoce do câncer infantojuvenil: responsabilidade de todos. Rev Assoc Med Bras. 2003;49(1):29-34. doi: https://dx.doi.org/10.1590/ S0104-42302003000100030.

11. Pollock MPH, Krischer JP, Vietti TJ. Interval between symptom onset and diagnosis of pediatric solid tumors. J Pediatr. 1991;119:725-32. doi: https://doi.org/10.1016/S00223476(05)80287-2.

12. Rodrigues KE, Camargo B, Ribeiro KB, Latorre MRDO. Interval between symptom onset and diagnosis on pediatric malignant disease. Analysis of 2975 cases. In: 32 nd SIOP Meeting, Amsterdam; 2000.

13. Brasil. Ministério da Saúde. Instituto Nacional de Câncer José Alencar Gomes da Silva (INCA). Registros Hospitalares de Câncer (RHC). Rio de Janeiro; 2017 [citado 11 out. 2018]. Disponível em: https://irhc.inca.gov.br/.

14. Chaffin VS. Análise dos registros hospitalares de câncer para o monitoramento do câncer infanto-juvenil [dissertação]. Rio de Janeiro: Fundação Oswaldo Cruz; 2016.

15. Costa, MAM, Magluta A, Gomes Junior SC. Evaluation of continuing education of family health strategy teams for the early identification of suspected cases of cancer in children. BMC Med Educ. 2017;17:155;2-6. doi: https://doi. org/10.1186/s12909-017-0993-1. 